\title{
Multi-subject Registration for Unbiased Statistical Atlas Construction
}

\author{
Mathieu De Craene ${ }^{1,2,3}$, Aloys du Bois d'Aische ${ }^{1,2,3}$, Benoît Macq ${ }^{1}$, and \\ Simon K. Warfield ${ }^{2,3}$ \\ 1 Communications and Remote Sensing Laboratory, Université catholique de \\ Louvain, Louvain-la-Neuve, BELGIUM. \\ 2 Surgical Planning Laboratory \\ 3 Computational Radiology Laboratory, Brigham and Women's Hospital, Harvard \\ Medical School, Boston MA, USA.
}

\begin{abstract}
This paper introduces a new similarity measure designed to bring a population of segmented subjects into alignment in a common coordinate system. Our metric aligns each subject with a hidden probabilistic model of the common spatial distribution of anatomical tissues, estimated using STAPLE. Our approach does not require the selection of a subject of the population as a "target subject", nor the identification of "stable" landmarks across subjects. Rather, the approach determines automatically from the data what the most consistent alignment of the joint data is, subject to the particular transformation family used to align the subjects. The computational cost of joint simultaneous registration of the population of subjects is small due to the use of an efficient gradient estimate used to solve the optimization transform aligning each subject. The efficacy of the approach in constructing an unbiased statistical atlas was demonstrated by carrying out joint alignment of 20 segmentations of MRI of healthy preterm infants, using an affine transformation model and a FEM volumetric tetrahedral mesh transformation model.
\end{abstract}

\section{Introduction}

Statistical atlases are an important representation for characterizing anatomy and anatomical variation. They have applications in characterizing normal anatomy, and in identifying structural differences between populations, such as between healthy newborn infants and infants with white matter injury. Furthermore, statistical atlases provide prior probability models useful to constrain segmentation and registration algorithms to ensure robust and rapid operation. For example, given prior information about the main modes of shape variation, a non-rigid registration algorithm may optimize initially upon these main modes of variation instead of considering all parameters of the deformation model simultaneously. The use of statistical atlases has also been investigated for improving the accuracy of automatic segmentations algorithms to extract data despite the noise and artifacts by capturing a priori information.

A statistical atlas is a model of the expected anatomy, usually represented as a probability of an anatomical structure or tissue being present at each voxel 
of a 3D lattice. Such an atlas may be constructed by sampling from a large population of subjects, identifying the anatomy or tissue present in each subject by segmentation and then projecting the segmentation into a common coordinate system. Previous approaches to do this have differed in the manner in which the appropriate alignment of the subjects is defined, and by the flexibility of the transformation aligning the subjects. For example, some have used manually selected landmarks [1] or intensity features [2]. As described in [2], a datadriven approach that avoids any bias introduced by selecting a set of landmarks is a desirable feature for next-generation statistical atlas construction algorithms. Furthermore, the bias introduced by selecting a particular subject (who has an anatomy of unknown typicality) and aligning further scans with that subject [4] is undesirable. Algorithms that attempt to undo this bias by removing an average transformation following the alignment to a single subject have been described (see [5] and [8]) but unfortunately require limiting assumptions regarding the accuracy of the individual subject-to-target alignment (see for example [8] note on page 1021) and in practice have generated statistical atlases that differ when different target subjects are selected.

We have previously investigated an approach to atlas construction that avoids the bias introduced by a single subject by carrying out simultaneous alignment of a collection of subjects 14. This approach was built upon previous work in handwriting recognition by alignment [6. This algorithm required the expensive construction of a large joint probability distribution. Bhatia[1] proposed to select one arbitrary image to act as an intensity reference, and then to add all pairs of intensities, comprising the voxel intensity in the reference and the corresponding intensity in each image, to the same joint histogram. We propose here a new algorithm, leveraging our recent work in the automated assessment of similarity of segmentations 14 to enable the construction of a statistical atlas by joint alignment of a collection of subjects. The new algorithm presented here is both efficient, in not requiring an unwieldy large joint probability distribution estimate, and avoids identifying any particular subject as a target and hence avoids any target bias. It does not require the identification of common and stable landmarks across subjects, but rather uses a data-driven approach to identify from the input data itself, the most consistent joint alignment of the subjects.

\section{Algorithm Description}

The purpose of the algorithm is to identify the best joint alignment of a collection of segmentations. This is done by solving for the set of transformations $\left\{T_{j}\right\}$ mapping each subject $j$ into a common coordinate system. The common coordinate system is also estimated from the aligned data. To speed up the convergence, the set of $\left\{T_{j}\right\}$ is initialized to map the center of gravity of each subject to the center of the common coordinate system. The algorithm is a generalized Expectation-Maximization algorithm, where the subject segmentations are the observed data, and hidden data is the anatomical label of each voxel of the statistical atlas. The parameters to be estimated are the transform for 
each subject that aligns each subject best with the hidden statistical atlas, and parameters that describe the similarity of the subject labels with that of the hidden statistical atlas. The similarity parameters are estimated as in our earlier work on validation of image segmentation, and can be interpreted as, for each subject label, the probability that the subject label will match the atlas label at the same voxel under the current transformation. A closed form expression for these parameters is straightforward to solve, whereas the transformation parameters have no closed form solution and must be identified by an optimization procedure. Therefore, the implementation iterates between these two steps:

- Expectation step: A probabilistic estimate of the statistical atlas labels is made given the set of subjects $1 \ldots J$ and the set of current transformations $\left\{T_{j}\right\}$.

- Maximization step: For each subject $j$, the transformation $T_{j}$ is updated to maximize the similarity of the subject with the probabilistic statistical atlas estimate computed in the first step. The parameters describing the consistency of each aligned subject to the probabilistic atlas are found by maximum likelihood estimation.

The entire procedure is then iterated to convergence, with the consistency parameter estimates being used to update the probabilistic atlas labels, which then allows new refined estimates of the simultaneous joint alignment of the subjects. As this constitutes a generalized Expectation-Maximization algorithm, convergence to a local optimum is guaranteed.

\subsection{Probabilistic Atlas Estimation}

In this step, a probability map for each label is computed over the whole atlas image domain using an iterative EM algorithm described in [13]14]. Rather than also updating estimates of the transformation parameters at each iteration, they are held fixed until the probabilistic atlas estimate is converged, and are only then updated. After convergence of the probabilistic atlas estimate, the probability to find the label $s$ at the voxel $i$ in the atlas can be written as

$$
\begin{aligned}
W_{s i} & =\frac{1}{\alpha} f\left(A_{i}=s\right) \prod_{j} f\left(D_{i j} \mid A_{i}=s, \hat{\boldsymbol{\Theta}}, T_{j}\right) \\
& =\frac{1}{\alpha} f\left(A_{i}=s\right) \prod_{j} \hat{\theta}_{D_{i j}^{j} s}
\end{aligned}
$$

where $\alpha$ is a normalization constant to ensure $\sum_{s} W_{s i}=1, f\left(A_{i}=s\right)$ is a prior probability on the ground label $A_{i}, D_{i j}$ is the value of the $i^{\text {th }}$ pixel in the $j^{\text {th }}$ subject. $\hat{\boldsymbol{\Theta}}$ is the set of matrix of parameters describing the similarity of each subject with the probabilistic atlas. For a subject $j$, each element of the matrix is $\hat{\theta}_{s^{\prime} s}^{j}$, the probability for observing the label $s^{\prime}$ when the ground truth is $s$ and can be computed from the set of $W_{s i}$ as: 


$$
\hat{\theta}_{s^{\prime} s}^{j}=\frac{1}{\beta} \sum_{i: D_{i j}=s^{\prime}} W_{s i}
$$

where $\beta$ is a normalization constant to get $\sum_{s^{\prime}} \hat{\theta}_{s^{\prime} s}^{j}=1$.

After convergence, the solutions of Equations 1 and 3 become stationary.

\subsection{Subject to Probabilistic Atlas Registration}

Once the probabilistic atlas $W_{s i}$ has been computed for each label $s$, at each position $i$ of the atlas image domain, the set of transformations $\left\{T_{j}\right\}$ is updated for each subject by solving the transformation $T_{j}$ maximizing the mean trace of the $j^{\text {th }}$ subject similarity matrix :

$$
\frac{1}{N_{s}} \sum_{s} \hat{\theta}_{s s}^{j}
$$

Since $\hat{\theta}_{s s}^{j}$ denotes the true positive fraction of the label $s$, this cost function seeks to align the subject $j$ to have a spatial distribution of labels as similar as possible to that of the probabilistic atlas.

To illustrate the behavior of this metric around the optimum, the mean trace of the specifity matrix for one subject is plotted around the optimum as a function of translations and rotations parameters in Figure 1 for variations in a $[-10,+10]$ interval.

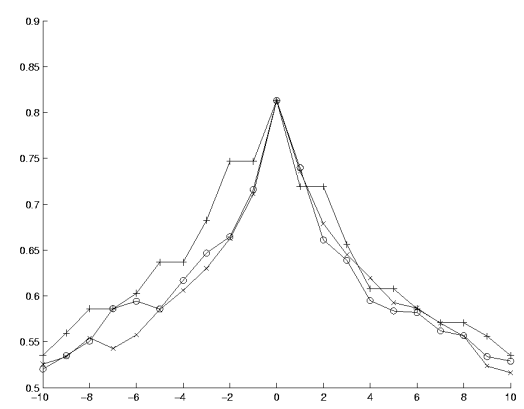

Translations $[\mathrm{mm}]: t_{x}(\mathrm{o}), t_{y}(\mathrm{x}), t_{z}(+)$

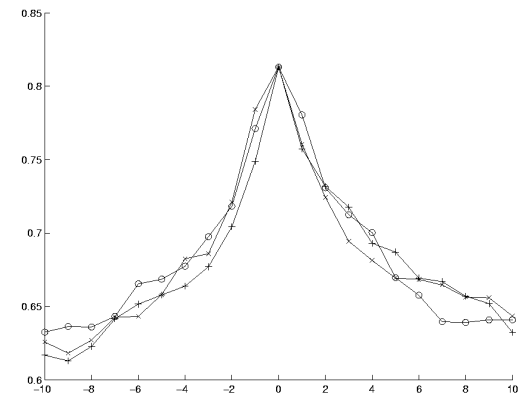

Rotations $[\mathrm{deg}]: r_{x}(\mathrm{o}), r_{y}(\mathrm{x}), r_{z}(+)$

Fig. 1. The cost function used for multi-subject registration is plotted for one subject around the optimum found by the algorithm as a function of the translations and rotations parameters.

\section{$3 \quad$ Experiments}

The 22 high resolution T1w, PDw and T2w MRI images of healthy preterm infants were segmented into tissue types (background, skin, sub-cortical and cortical gray matter, cerebrospinal fluid, myelinated white matter, unmyelinated 
white matter) using an intensity- and atlas- based optimal classifier as previously described and validated [12]. For this experiment, these labels have been gathered in four labels: background, unmyelinated white matter, basal ganglia and a class containing the remaining brain voxels.

The set of transformations mapping the atlas space on each subject was composed of a rigid and scale transformation followed by an FEM based non-rigid transformation. The process has been initialized by a set of translations sending the center of the atlas to the center of gravity of each subject using the itk: : ImageMomentsCalculator class. This is done to improve the initialization and therefore reduce the computation time. The sum after bringing the center of the atlas to the center of gravity of each subject is plotted in Figure 3(a). The metric described in section 2.2 has been optimized using an iterative gradient ascent scheme. The gradient ascent is based on the Simultaneous Stochastic Perturbation Approximation (SPSA) of the gradient, firstly introduced by Spall [10]. The SPSA method incorporates two gains decreasing with the number of iterations shrinking the distance over which the finite differences are calculated as well as the learning rate.This method relies only on two measurements of the objective function to be optimized. The SPSA optimization routine has been integrated into the ITK $[9]$ optimization framework.

\subsection{Rigid and Scale Transformation Model}

The algorithm described in Section 2 has been run using a transformation model allowing translations, rotations and three scale factors. After registration of each subject on the first estimate of the probabilistic atlas (to improve the consistency of each subject as described in Section 2.2) the probabilistic atlas (Ground Truth estimate) has been recomputed and plotted in Figure 2. The process is then iterated until convergence of the whole set of parameters (three iterations of probabilistic atlas estimation and registration parameters estimation were needed in this case). The sum of all subjects after rigid and scale alignment is plotted in Figure 3(b).

\subsection{FEM Volumetric Tetrahedral Mesh Transformation Model}

To refine the results obtained in the former section, a volumetric tetrahedral mesh has been used to extend the transformation model. The transformation is then parameterized by the displacements at each node of the mesh. The nodes displacements are propagated to any point of the common space of coordinates using the shape functions of the element containing this point.

$$
u_{l}(x)=\sum_{n \in \text { Nodes }} v_{l}^{n} N^{n}(x)
$$

where $u_{l}$ stands for the $l^{\text {th }}$ component of the displacement field, $x$ is the $3 \mathrm{D}$ position in the fixed image volume, $v_{l}^{n}$ is the displacement of the $n^{\text {th }}$ node of the element and $N^{n}(x)$ is the shape function associated to the node $n$. In this 
experiment, a coarse mesh has been obtained from a $64 \times 64 \times 10$ voxels $^{3}$ BCC grid as described in [7]. To improve the convergence of the optimization process, we reduce the dimensionality of the space of parameters by selecting at each iteration a set of "active nodes". For this selection, a first derivative is computed by generating a large number of gradient estimates using stochastic perturbations on the whole set of nodes. The nodes where the norm of the derivative is above a fixed threshold are selected as active nodes. Active nodes displacements are propagated to other nodes by adding in the cost function a linear elastic regularization constrain as we describe in 3 . The sum of all subjects after FEM alignment of each subject on the probability map (starting from the results of section 3.1) is plotted in Figure 3(c). The border of the brain has become more contrasted after FEM alignment. Figure 3 (d) plots a weighted sum of the four labels in all subjects after FEM alignment (the intensity at voxel $i$ is equal to $\left.\sum_{j} D_{i j} W_{D_{i j}, s}\right)$.

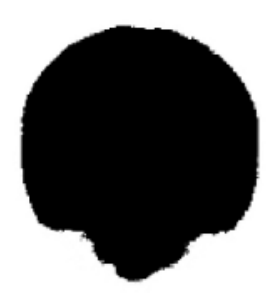

(a)

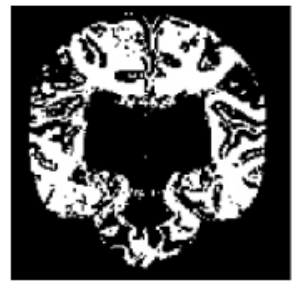

(b)

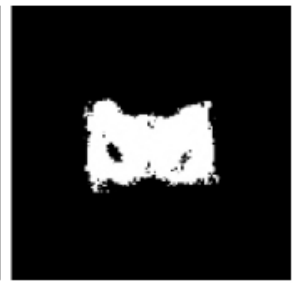

(c)

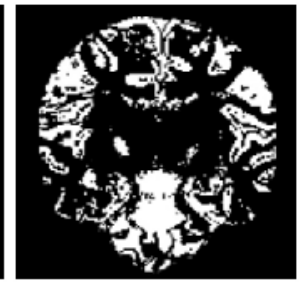

(d)

Fig. 2. The STAPLE ground truth probability for each label is plotted after 1 iteration of our algorithm for the background (a), the unmyelinated white matter (b), the basal ganglia(c) and the remaining brain voxels classified in a separate class (d). The steep rising from regions where the probability is 0 (black voxels) to regions where the probability is 1 results from the important weight give to consistent subjects in the $W_{\text {si }}$ computation.

\section{Conclusions and Future Work}

This study, carried out on 22 preterm healthy infants, has demonstrated that the STAPLE estimate of a probability map for each label can be used to align a collection of subjects to a common space of coordinates. The use of volumetric adaptive FEM meshes could allow the refinement of the transformation model in specific areas, splitting elements where a significant variability is observed for the current alignment. Statistical analysis of the set of node displacements will allow to include prior information in non-rigid registration algorithms by seeking first to optimize the main deformations modes observed in the atlas. 


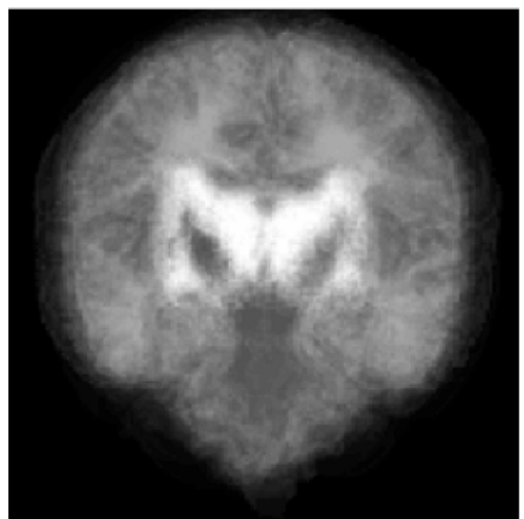

(a) Center of gravity alignment

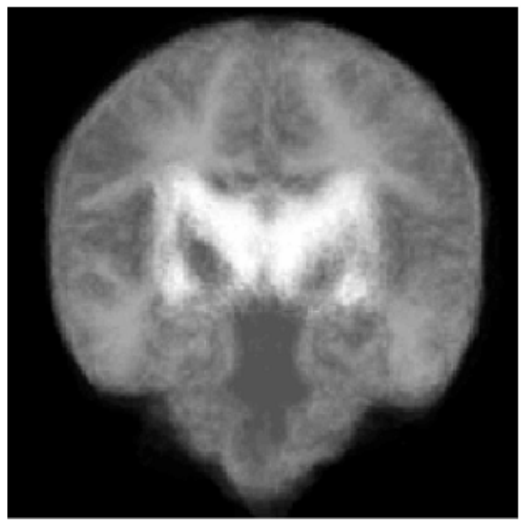

(c) F'EM alignment

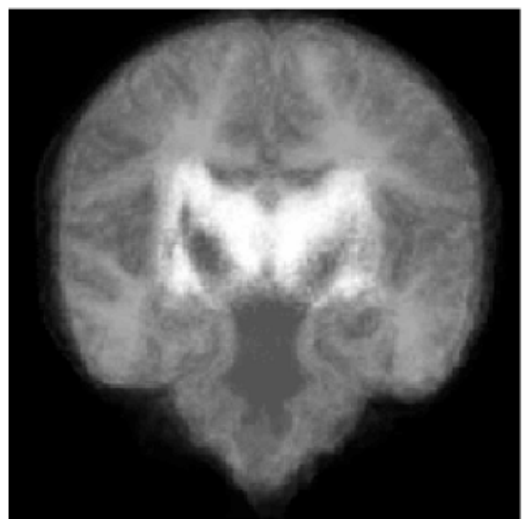

(b) Rigid and scale alignment

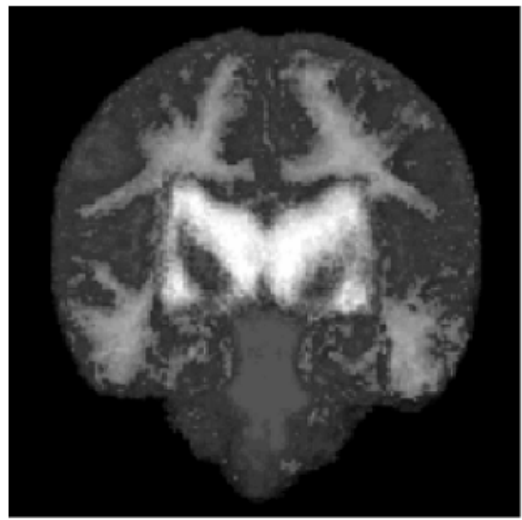

(d) Weighted sum after alignment

Fig. 3. Sub-figure (a) plots the sum of all 22 pre-term healthy subjects after bringing the center of gravity of each subject to the center of the atlas image. Sub-figure (b) shows the sum of all subjects after alignment to the STAPLE probabilistic atlas (plotted in Figure 2] using translations, rotations around $X, Y$ and $Z$ axis, and 3 scale factors. A simple non-rigid transformation model using a regular FEM tetrahedral mesh has been used to improve the accuracy around the borders of the brain. The result is plotted in sub-figure (c). The FEM mesh has been built on a $64 \times 64 \times 10$ voxels $^{3}$ BCC regular grid. Sub-figure (d) plots a weighted - by the tissue probability in the statistical atlas - combination of labels for all subjects after FEM alignment.

Acknowledgments. Mathieu De Craene and Aloys du Bois d'Aische are working towards a Ph.D. degree with a grant from the Belgian FRIA. This investigation was supported in part by the Region Wallone Mercator project, NIH grants P41 RR13218, NIH P01 CA67165, NIH R01 RR11747 and NIH R01 AG19513 and by a research grant from the Whitaker Foundation. We thank Dr. Terrie Inder for the neonate MRI data used in this study. 


\section{References}

1. K.K Bhatia, J.V. Hajnal, B.K. Puri, A.D. Edwards, and D. Rueckert. Consistent Groupwise Non-Rigid Registration for Atlas Construction. In ISBI, 2004.

2. D. L. Collins. 3D Model-based segmentation of individual brain structures from magnetic resonance imaging data. PhD thesis, McGill University, Montreal, 1994.

3. Mathieu De Craene, Aloys du Bois d'Aische, Ion-Florin Talos, Matthieu Ferrant, Peter McL. Black, Ferenc Jolesz, Ron Kikinis, Benoit Macq, and Simon K. Warfield. Dense Deformation Field Estimation for Brain Intra-Operative Images Registration. In SPIE Medical imaging : Image Processing, 2004.

4. PF. D' Haese, V. Duay, B. Macq, T. Merchant, and Dawant B. Atlas-based segmentation of the brain for 3-dimensional treatment planning in children with infratentorial ependymoma. In Sixth International Conference on Medical Image Computing and Computer-Assisted Intervention. Springer-Verlag, 2003.

5. A. Guimond, J. Meunier, and J.-P. Thirion. Average brain models: A convergence study. Computer Vision and Image Understanding, 77(2):192-210, 1999.

6. Erik G. Miller. Learning from One Example in Machine Vision by Sharing Densities. PhD thesis, EECS, MIT, Boston, 2002.

7. N. Molino, R. Bridson, J. Teran, and R. Fedkiw. A crystalline, red green strategy for meshing highly deformable objects with tetrahedra. In 12th International Meshing Roundtable, pages 103-114, Sandia National Laboratories, september 2003.

8. D. Rueckert, A. F. Frangi, and J. A. Schnabel. Automatic construction of 3$\mathrm{d}$ statistical deformation models of the brain using nonrigid registration. IEEE Trans Med Imaging, 22(8):1014-25, 2003.

9. Luis Ibanez Will Schroeder. The ITK Software Guide. The Insight Consortium.

10. J. C. Spall. Overview of the simultaneous perturbation method for efficient optimization. http://techdigest. jhuapl.edu/td/td1904/spall.pdf, Hopkins APL Technical Digest, 19:482-492, 1998.

11. J. Talairach and P. Tournoux. Co-planar stereotaxic atlas of the human brain: 3-dimensional proportional system - an approach to cerebral imaging. New York, NY: Thieme Medical Publishers, 1998.

12. Simon K. Warfield, Michael Kaus, Ferenc A. Jolesz, and Ron Kikinis. Adaptive, Template Moderated, Spatially Varying Statistical Classification. Med Image Anal, 4(1):43-55, Mar 2000.

13. Simon K. Warfield, K. H. Zou, and III. W. M. Wells. Validation of image segmentation and expert quality with an expectation-maximization algorithm. In Fifth International Conference on Medical Image Computing and Computer-Assisted Intervention. Springer-Verlag, 2002.

14. Simon K. Warfield, K. H. Zou, and W. M. Wells. Simultaneous truth and performance level estimation (staple): An algorithm for the validation of image segmentation. IEEE Trans Med Imag, 2004. Accepted to appear. 